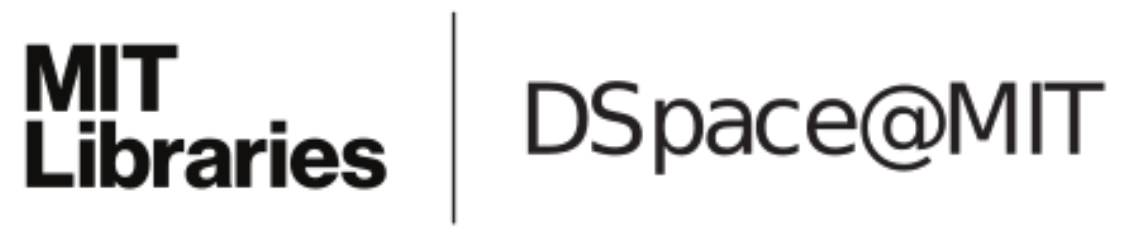

\author{
MIT Open Access Articles
}

Inhomogeneous phases in the Nambu-
Jona-Lasinio and quark-meson model

The MIT Faculty has made this article openly available. Please share how this access benefits you. Your story matters.

Citation: Nickel, Dominik . "Inhomogeneous phases in the Nambu- Jona-Lasinio and quarkmeson model." Physical Review D 80.7 (2009): 074025. (c) 2009 The American Physical Society

As Published: http://dx.doi.org/10.1103/PhysRevD.80.074025

Publisher: American Physical Society

Persistent URL: http://hdl.handle.net/1721.1/52505

Version: Final published version: final published article, as it appeared in a journal, conference proceedings, or other formally published context

Terms of Use: Article is made available in accordance with the publisher's policy and may be subject to US copyright law. Please refer to the publisher's site for terms of use. 


\title{
Inhomogeneous phases in the Nambu-Jona-Lasinio and quark-meson model
}

\author{
Dominik Nickel \\ Center for Theoretical Physics, Massachusetts Institute of Technology, Cambridge, Massachusetts 02139, USA
}

(Received 10 July 2009; published 22 October 2009)

\begin{abstract}
We discuss inhomogeneous ground states of the Nambu-Jona-Lasinio and quark-meson model within mean-field approximation and their possible existence in the respective phase diagrams. For this purpose we focus on lower-dimensional modulations and point out that known solutions in the $2+1$ and $1+1$ dimensional (chiral) Gross-Neveu model can be lifted to the to the $3+1$ dimensional Nambu-JonaLasinio model. This is worked out in detail for one-dimensional modulations and numerical results for the phase diagrams are presented. Focus is put on the critical point and on vanishing temperatures. As an interesting result the first order transition line in the phase diagram of homogeneous phases gets replaced by an inhomogeneous phase which is bordered by two second order transition lines.
\end{abstract}

DOI: 10.1103/PhysRevD.80.074025

\section{INTRODUCTION}

Until today the phase diagram of quantum chromodynamics (QCD) is subject to intense theoretical and experimental investigations (for dedicated reviews see Ref. [1]). Experimentally its structure at finite temperatures is explored by heavy ion collisions, which currently focus on the formation and properties of a strongly interacting plasma at large temperatures as well as on the search for a chiral critical point in the phase diagram. Theoretically ab initio calculations are limited to small net-baryon densities and as a consequence possible scenarios at nonvanishing densities and strong coupling are often discussed within phenomenological models.

Since Nambu-Jona-Lasinio (NJL)-type models ${ }^{1}$ share global symmetries and the phenomenon of chiral symmetry breaking with QCD, they are-typically in mean-field approximation - widely used to study the phase diagram at moderate temperatures and densities. In this context, e.g. color superconductivity $[1,2]$ as well as the location of the critical point(s) have been addressed [3]. In recent years this branch of models has been extended to Polyakov-NJLtype models, which include the dynamics of the Polyakovloop as the order parameter of confinement [4-6]. However for vanishing temperatures these models reduce to the respective NJL-type model.

In this work we investigate inhomogeneous ground states in the NJL [7] and QM model [8]. These are characterized by a spatially varying order parameter and have been discussed for QCD at least in the large $N$ limit, where they are expected to form the ground state at sufficiently high densities [9-11]. Related to this they show up in holographic models [12] and in the quarkyonic matter picture [13] that suggests a similar structure for QCD.

\footnotetext{
${ }^{1}$ We refer to NJL-type models as models that at least in the applied approximation reduce to the NJL model, possibly extended by additional pointlike interactions, on a technical level. This includes, e.g. simplified ansätze for the gluon interaction, the use of different regularizations, the instanton liquid model and the quark-meson (QM) model.
}

PACS numbers: 12.38.Mh, 21.65.Qr, 25.75.Nq

The investigations of these phases is however limited, mainly because they are technically much more involved. Within the NJL model such phases have been analyzed at vanishing temperatures applying further truncations [14] as well as for the so-called chiral density wave $[15,16]$. In the latter case, the order parameter is assumed to be a plane wave and can be solved on mean-field level for vanishing current quark masses. In addition, it has been suggested recently that the first order phase transition in the phase diagram of the NJL model, at least in the vicinity of the chiral critical point, is replaced by two second order phase transition lines that border an inhomogeneous phase [17]. The intersection of the two lines defines a Lifshitz point, which coincides with the critical point in the NJL model.

Inhomogeneous phases have also been investigated in the $1+1$ dimensional (chiral) Gross-Neveu (GN) model ${ }^{2}$ for the large $N$ limit, which technically corresponds to a mean-field approximation. Not only the understanding of the phase diagram has been improved significantly as it possesses regions with inhomogeneous ground states [1820], but also theoretical understanding of the integrability of these models has been obtained. In the chiral limit all self-consistent solutions can in principle be classified [21] and the phase diagrams have been analyzed in detail [22]. For the case of the GN model it is furthermore possible to introduce finite quark masses and to study their effect on the structure of the phase diagram [19].

The underlying idea of the present investigation is based on the observation that the theoretical problem of finding self-consistent inhomogeneous phases with a lowerdimensional modulation can be reduced to a problem in a lower-dimensional model. This is mainly due to the structure of the mean-field Hamiltonian, which is of Dirac type. $^{3}$ As a consequence self-consistent phases with a

\footnotetext{
${ }^{2}$ The chiral GN model is also called the $1+1$ dimensional NJL model. The global $Z_{2}$ symmetry of the GN model is extended to a $U(1)$ symmetry in this model.

${ }^{3}$ For the $2+1$ dimensional GN model a similar approach has been pursued in Ref. [23].
} 
one-dimensional modulation in the NJL model can be studied on similar grounds as in the $1+1$ dimensional (chiral) GN model and all results obtained for the latter can be used here. In particular, all self-consistent inhomogeneous solutions with a one-dimensional modulation are in principle also known for the NJL model, at least in the chiral limit. Furthermore we can investigate self-consistent inhomogeneous phases at finite quark masses, which has not been possible before. The considered inhomogeneous ground states are then lattices of domain-wall solitons. In the same way self-consistent phases with a twodimensional modulation could be related to those in the $2+1$ dimensional GN model. However, no analytical solutions for a real two-dimensional modulation are known for the latter.

Being able to investigate the role of inhomogeneous phases in the phase diagram of the NJL model, we can confirm the picture obtained in Ref. [17] for the vicinity of the critical point and the absence of a first order phase transition line in the phase diagram. ${ }^{4}$ Furthermore, we can analyze the relation between inhomogeneous phases and, e.g. the strength of the first order phase transition (present in the case of homogeneous phases). For the main part of the paper we do not allow for pseudoscalar condensates, especially since only without those self-consistent solutions for finite current quark masses are known. We will however also discuss why these condensates are not expected for the ground state.

At the end of the paper we will finally also consider the QM model. The purpose of this is twofold: On the one hand, we would like to extend the analysis to a larger class of models in general; on the other hand, the regularization of the NJL model for inhomogeneous phases is nontrivial with regard to a combined vacuum and QCD phase diagram phenomenology.

The paper is organized as follows: In Sec. II we introduce the NJL model and the applied mean-field approximation allowing for inhomogeneous phases. After this we show in Sec. III how self-consistent solutions for lowerdimensional modulations can be obtained from lowerdimensional models. We then summarize self-consistent solutions of the (chiral) GN model in Sec. IV and work out in Sec. V how those can be used for the NJL model. Before coming to the QM model in Sec. VII, we remark in Sec. VI why pseudoscalar condensates are not expected. Finally, we discuss various numerical results in Sec. VIII and close with a summary and outlook in Sec. IX.

\section{NJL MODEL AND MEAN-FIELD APPROXIMATION}

In this work we concentrate on the two-flavor NJL model given by the Lagrangian

\footnotetext{
${ }^{4}$ This picture could of course be modified by the inclusion of color-superconducting phases.
}

$$
\mathcal{L}=\bar{\psi}\left(i \gamma^{\mu} \partial_{\mu}-\hat{m}\right) \psi+G_{s}\left((\bar{\psi} \psi)^{2}+\left(\bar{\psi} i \gamma^{5} \tau^{a} \psi\right)^{2}\right),
$$

where $\psi$ is the $4 N_{f} N_{c}$-dimensional quark spinor for $N_{f}=$ 2 flavors and $N_{c}=3$ colors, $\gamma^{\mu}$ are Dirac matrices, $G_{s}$ is the scalar coupling and $\hat{m}$ the mass matrix for degenerate quarks with current quark mass $m$. For $N_{f}=2$ the matrices $\tau^{a}$ are the conventional Pauli matrices.

We address phases with nonvanishing expectation values $\langle\bar{\psi} \psi\rangle=S(\mathbf{x})$ and $\left\langle\bar{\psi} i \gamma^{5} \tau^{a} \psi\right\rangle=P_{a}(\mathbf{x})$. Expanding bilinears around those expectation values and neglecting quadratic contributions, we then work within mean-field approximation. For technical reasons that will become more apparent in the following we furthermore restrict ourselves to the case where the direction of the vector $P_{a}(\mathbf{x})$ is constant in flavor space so that we can choose a flavor basis where $P_{1}(\mathbf{x})=P_{2}(\mathbf{x})=0$ and $P_{3}(\mathbf{x})=P(\mathbf{x})$. The mean-field Lagrangian therefore takes the form

$$
\begin{aligned}
\mathcal{L}_{\mathrm{MF}}= & \bar{\psi}\left(i \gamma^{\mu} \partial_{\mu}-m+2 G_{s} S(\mathbf{x})+2 i G_{s} \gamma^{5} \tau^{3} P(\mathbf{x})\right) \psi \\
& -G_{s}\left(S(\mathbf{x})^{2}+P(\mathbf{x})^{2}\right) .
\end{aligned}
$$

Since the mean-field Lagrangian is bilinear, the thermodynamic potential as an effective action in the expectation values can be formally evaluated. In the case of a periodic condensate with Wigner-Seitz cell $V$ and using the imaginary-time formalism (see e.g. Ref. [1]), the thermodynamic potential as an effective action in $S(\mathbf{x}), P(\mathbf{x})$ is then given by

$$
\begin{aligned}
\Omega(T, \mu ; S(\mathbf{x}), P(\mathbf{x}))= & -\frac{T}{V} \ln \int \mathcal{D} \bar{\psi} \mathcal{D} \psi \exp \\
& \times\left(\int_{x \in[0,(1 / T)] \times V}\left(\mathcal{L}_{\mathrm{MF}}+\mu \bar{\psi} \gamma^{0} \psi\right)\right) \\
= & -\frac{T N_{c}}{V} \sum_{n} \operatorname{Tr}_{D, f, V} \\
& \times \log \left(\frac{1}{T}\left(i \omega_{n}+\tilde{H}_{\mathrm{MF}}-\mu\right)\right) \\
& +\frac{G_{s}}{V} \int_{V}\left(S(\mathbf{x})^{2}+P(\mathbf{x})^{2}\right),
\end{aligned}
$$

where the functional logarithm and trace act on Dirac, color, and coordinate space. The Hamiltonian $\tilde{H}_{\mathrm{MF}}$ is obtained from Eq. (2) and reads

$$
\tilde{H}_{\mathrm{MF}}=-i \gamma^{0} \gamma^{i} \partial_{i}+\gamma^{0}\left(m-2 G_{s} S(\mathbf{x})-2 i G_{s} \gamma^{5} \tau^{3} P(\mathbf{x})\right),
$$

which is a direct product of the two isospectral Hamiltonians

$$
H_{\mathrm{MF}, \pm}=-i \gamma^{0} \gamma^{i} \partial_{i}+\gamma^{0}\left(m-2 G_{s} S(\mathbf{x}) \mp 2 i G_{s} \gamma^{5} P(\mathbf{x})\right) .
$$

Assuming a sensible regularization for the functional trace we can then express the thermodynamic potential through 
the eigenvalues $\left\{E_{n}\right\}$ of $H_{\mathrm{MF},+}$ as

$$
\begin{aligned}
\Omega(T, \mu ; M(\mathbf{x}))= & -\frac{2 T N_{c}}{V} \sum_{E_{n}} \ln \left(2 \cosh \left(\frac{E_{n}-\mu}{2 T}\right)\right) \\
& +\frac{1}{V} \int_{V} \frac{|M(\mathbf{x})-m|^{2}}{4 G_{s}}+\text { const, }
\end{aligned}
$$

where $M(\mathbf{x})=m-2 G_{s}(S(\mathbf{x})+i P(\mathbf{x}))$. We note that the mass function $M(\mathbf{x})$ as the order parameter can be complex and parameterizes a $U(1)$ subgroup.

In order to minimize the thermodynamic potential in the order parameter $M(\mathbf{x})$ we can address the stationary constraint $\frac{\delta \Omega}{\delta M(\mathbf{x})^{*}}=0$, which in turn gives the gap equation

$$
\begin{aligned}
M(\mathbf{x})= & m+\frac{4 G_{s} N_{c}}{V} \sum_{E_{n}} \tanh \left(\frac{E_{n}-\mu}{2 T}\right) \\
& \times \psi_{n}^{\dagger}(\mathbf{x}) \frac{\partial H_{\mathrm{MF},+}}{\partial M(\mathbf{x})^{*}} \psi_{n}(\mathbf{x}) \\
= & m+\frac{2 G_{s} N_{c}}{V} \sum_{E_{n}} \tanh \left(\frac{E_{n}-\mu}{2 T}\right) \\
& \times \bar{\psi}_{n}(\mathbf{x})\left(1-\gamma^{5}\right) \psi_{n}(\mathbf{x})
\end{aligned}
$$

as a self-consistency condition on $M(\mathbf{x})$. Here, $\psi_{n}(\mathbf{x})$ are the normalized eigenvectors of the Hamiltonian for the eigenvalues $E_{n}$, i.e. $\frac{1}{V} \int_{V} \psi_{n}^{\dagger} \psi_{n}=1$.

\section{LOWER-DIMENSIONAL MODULATIONS}

Having the formal expressions for thermodynamic potential and gap equation, we turn toward inhomogeneous phases. The focus is the determination of the eigensystem of a Hamiltonian and the check of self-consistency. As will be discussed in the following, these problems can be dimensionally reduced for lower-dimensional modulations with the help of Lorentz symmetry.

Suppose we have a representation $S(\Lambda)$ for the Lorentz transformation $\Lambda$ that acts on the momentum operator of free spinors $P^{\mu}=\left(H, P_{i}\right)^{\mu}$ as

$$
S(\Lambda) P^{\mu} S^{-1}(\Lambda)=\Lambda_{\nu}^{\mu} P^{\nu} .
$$

If the system is translationally invariant in one or more directions, the corresponding momenta $P_{\perp}$ commute with the Hamiltonian $H$ and we can label its eigenstates also by $\mathbf{p}_{\perp}$, the eigenvalue of $P_{\perp}$. Now take $\psi_{\lambda, 0}$ to be the eigenvector with $H \psi_{\lambda, 0}=\lambda \psi_{\lambda, \mathbf{0}}$ and $P_{\perp} \psi_{\lambda, \mathbf{0}}=0, \Lambda^{\mu}{ }_{\nu}$ to be the Lorentz transformation that boosts $(\lambda, \mathbf{0})^{\mu}$ to $\left(\lambda \sqrt{1+\mathbf{p}_{\perp}^{2} / \lambda^{2}}, \mathbf{p}_{\perp}\right)^{\mu}$ and define

$$
\psi_{\lambda \sqrt{1+\mathbf{p}_{\perp}^{2} / \lambda^{2}, \mathbf{p}_{\perp}}}=\left(\sqrt{1+\mathbf{p}_{\perp}^{2} / \lambda^{2}}\right)^{-1 / 2} S^{-1}(\Lambda) \psi_{\lambda, \mathbf{0}} .
$$

The prefactor implements the proper normalization for the conventional choice of $S(\Lambda)$ [24] and it is straightforward to check that
$P^{\mu} \psi_{\lambda \sqrt{1+\mathbf{p}_{\perp}^{2} / \lambda^{2}}, \mathbf{p}_{\perp}}=\left(\lambda \sqrt{1+\mathbf{p}_{\perp}^{2} / \lambda^{2}}, \mathbf{p}_{\perp}\right)^{\mu} \psi_{\lambda \sqrt{1+\mathbf{p}_{\perp}^{2} / \lambda^{2}}, \mathbf{p}_{\perp}}$.

Therefore, the whole eigenvalue spectrum can be constructed from the subspace spanned by $\left\{\psi_{\lambda, 0}\right\}$, and for the thermodynamic potential we obtain

$$
\begin{aligned}
\Omega(T, \mu ; M(\mathbf{x}))= & -\frac{2 T N_{c}}{V_{\|}} \sum_{\lambda} \int \frac{d \mathbf{p}_{\perp}}{(2 \pi)^{d_{\perp}}} \\
& \times \ln \left(2 \cosh \left(\frac{\lambda \sqrt{1+\mathbf{p}_{\perp}^{2} / \lambda^{2}}-\mu}{2 T}\right)\right) \\
& +\frac{1}{V} \int_{V} \frac{|M(\mathbf{x})-m|^{2}}{4 G_{s}}+\text { const, }
\end{aligned}
$$

where $V_{\|}$is a Wigner-Seitz cell of the lower-dimensional modulation. Addressing the self-consistency condition in Eq. (7) we note that $S(\mathbf{x})$ and $P(\mathbf{x})$ are scalar and pseudoscalar, respectively. We find

$$
\begin{aligned}
\bar{\psi}_{\lambda \sqrt{1+\mathbf{p}_{\perp}^{2} / \lambda^{2}}, \mathbf{p}_{\perp}} \psi_{\lambda \sqrt{1+\mathbf{p}_{\perp}^{2} / \lambda^{2}}, \mathbf{p}_{\perp}} & =\frac{\lambda}{\sqrt{\lambda^{2}+\mathbf{p}_{\perp}^{2}}} \bar{\psi}_{\lambda, \mathbf{0}} \psi_{\lambda, \mathbf{0}}, \\
\bar{\psi}_{\lambda \sqrt{1+\mathbf{p}_{\perp}^{2} / \lambda^{2}}, \mathbf{p}_{\perp}} \gamma^{5} \psi_{\lambda \sqrt{1+\mathbf{p}_{\perp}^{2} / \lambda^{2}}, \mathbf{p}_{\perp}} & =\frac{\lambda}{\sqrt{\lambda^{2}+\mathbf{p}_{\perp}^{2}}} \bar{\psi}_{\lambda, \mathbf{0}} \gamma^{5} \psi_{\lambda, \mathbf{0}},
\end{aligned}
$$

and the gap-equation takes the form

$$
\begin{aligned}
M(\mathbf{x})= & m+\frac{2 G_{s} N_{c}}{V_{\|}} \sum_{\lambda} \int \frac{d \mathbf{p}_{\perp}}{(2 \pi)^{d_{\perp}}} \tanh \left(\frac{\lambda \sqrt{1+\mathbf{p}_{\perp}^{2} / \lambda^{2}}-\mu}{2 T}\right) \\
& \times \frac{\lambda}{\sqrt{\lambda^{2}+\mathbf{p}_{\perp}^{2}}} \bar{\psi}_{\lambda, \mathbf{0}}\left(1-\gamma^{5}\right) \psi_{\lambda, \mathbf{0}} .
\end{aligned}
$$

Therefore, we have formal expressions for the thermodynamic potential and the gap equation in terms of the subspace spanned by the eigensystem corresponding to $\mathbf{p}_{\perp}=0$. To be more specific we now choose the Weyl representation for the $\gamma$ matrices, i.e.

$$
\gamma^{0}=\left(\begin{array}{ll}
0 & \mathbf{1} \\
\mathbf{1} & 0
\end{array}\right), \quad \gamma^{i}=\left(\begin{array}{cc}
0 & \sigma^{i} \\
-\sigma^{i} & 0
\end{array}\right), \quad \gamma^{5}=\left(\begin{array}{cc}
-\mathbf{1} & 0 \\
0 & \mathbf{1}
\end{array}\right)
$$

with the conventional Pauli matrices $\sigma^{i}$ so that the Hamiltonian in Eq. (5) takes the form

$$
H_{\mathrm{MF},+}=\left(\begin{array}{cc}
i \sigma^{i} \partial_{i} & M(\mathbf{x}) \\
M(\mathbf{x})^{*} & -i \sigma^{i} \partial_{i}
\end{array}\right) .
$$

First restricting to a one-dimensional modulation in the $z$ direction, the Hamiltonian in the subspace corresponding to $\mathbf{p}_{\perp}=0$ is 


$$
H_{\mathrm{MF} ; 1 D}=\left(\begin{array}{cccc}
i \partial_{z} & & M(z) & \\
M(z)^{*} & -i \partial_{z} & & M(z) \\
& M(z)^{*} & -i \partial_{z} & i \partial_{z}
\end{array}\right),
$$

which can be block diagonalized into

$$
H_{\mathrm{MF} ; 1 D}^{\prime}=\left(\begin{array}{ll}
H_{1 D}(M(z)) & \\
& H_{1 D}\left(M(z)^{*}\right)
\end{array}\right),
$$

where

$$
H_{1 D}(M(z))=\left(\begin{array}{cc}
-i \partial_{z} & M(z) \\
M(z)^{*} & i \partial_{z}
\end{array}\right)
$$

The problem is therefore reduced to finding the quasiparticle spectrum of $H_{1 D}$, which is the Hamilton of the chiral GN model and which will be discussed in more detail in Sec. IV.

Considering the scenario with a two-dimensional modulation it is more convenient to assume the system to be translationally invariant in the $z$ direction. The Hamiltonian in Eq. (15) for $\mathbf{p}_{\perp}=0$ is then given by

$$
H_{\mathrm{MF} ; 2 D}=\left(\begin{array}{cccc} 
& i \partial_{x}-\partial_{y} & M(x, y) & \\
i \partial_{x}+\partial_{y} & & & M(x, y) \\
M(x, y)^{*} & & -i \partial_{x}+\partial_{y}
\end{array}\right) .
$$

This can be block diagonalized only for real $M(x, y)$, i.e. for $P(\mathbf{x})=0$. In this case we can cast the problem into the form

$$
\tilde{H}_{\mathrm{MF} ; 2 D}=\left(\begin{array}{cccc}
-M(x, y) & i \partial_{x}+\partial_{y} & & \\
i \partial_{x}-\partial_{y} & -M(x, y) & & \\
& & M(x, y) & i \partial_{x}+\partial_{y} \\
& & i \partial_{x}-\partial_{y} & M(x, y)
\end{array}\right),
$$

which corresponds to the GN model in two dimensions. The fact that we need a real mass function $M(x, y)$ is not surprising since there is no chiral GN model with twocomponent spinors in two dimensions.

In general, we are therefore able to constrain ourselves to a lower-dimensional model if we want to determine the quasiparticle spectrum for an inhomogeneous phase with a lower-dimensional modulation. However, a self-consistent modulation in the lower-dimensional model does not necessarily have to be self-consistent in the higherdimensional model. For the cases considered in this paper and essentially all analytical solutions known this will nevertheless be the case since for each eigenvector separately we will have

$$
\bar{\psi}_{\lambda, \mathbf{0}}\left(1-\gamma^{5}\right) \psi_{\lambda, \mathbf{0}} \propto a+b M(z),
$$

and there are enough parameters in the analytical expres- sion for the modulation to tune the coefficients $a, b$ such that the solution is self-consistent.

\section{INHOMOGENEOUS PHASES IN ONE-DIMENSIONAL MODELS}

Having realized that self-consistent solutions with lower-dimensional modulations may be obtained from lower-dimensional models, we now present some selfconsistent phases with a one-dimensional modulation. This reduces to the study of the (chiral) GN model whose Hamiltonian is given by Eq. (18), and in the following we summarize some results deduced from Refs. [18,19,21,25].

The thermodynamic potential can be evaluated by the knowledge of the density of states $\rho(\lambda)$, which enables us to perform the sum over the eigenvalue spectrum: For an arbitrary function $f(\lambda)$ it is given by

$$
\frac{1}{V_{\|}} \sum_{\lambda} f(\lambda)=\int d \lambda \rho(\lambda) f(\lambda)
$$

Recent investigations have shown that at least for the chiral limit all self-consistent solutions can be classified and that all information about the inhomogeneous phase is encoded in the band-edge energies $\left\{E_{i}\right\}$ of the valence bands of the quasiparticles [21]. For the case of one gapped valence band the general solution for a complex order parameter $M(z)$ has been determined [25] and the spectral density associated with the Hamiltonian $H_{1 D}$ is given by

$$
\rho(\lambda)= \begin{cases}\frac{1}{2 \pi} \frac{a(\lambda)+\left\langle|M(z)|^{2}\right\rangle}{\sqrt{\Pi_{j=1}^{4}\left(\lambda-E_{j}\right)}}, & \Pi_{j=1}^{4}\left(\lambda-E_{j}\right)>0 \\ 0, & \text { else }\end{cases}
$$

where

$$
\begin{aligned}
a(\lambda)= & 2\left(\lambda-\frac{1}{4} \sum_{j=1}^{4} E_{j}\right)^{2}-\frac{1}{8} \sum_{i<j}\left(E_{i}-E_{j}\right)^{2}, \\
\left\langle|M(z)|^{2}\right\rangle= & \frac{1}{4}\left(E_{1}+E_{2}-E_{3}-E_{4}\right)^{2} \\
& -\frac{\left(E_{1}-E_{3}\right)\left(E_{2}-E_{4}\right) \mathbf{E}\left(\frac{\left(E_{1}-E_{2}\right)\left(E_{3}-E_{4}\right)}{\left(E_{1}-E_{3}\right)\left(E_{2}-E_{4}\right)}\right)}{\mathbf{K}\left(\frac{\left(E_{1}-E_{2}\right)\left(E_{3}-E_{4}\right)}{\left(E_{1}-E_{3}\right)\left(E_{2}-E_{4}\right)}\right)}
\end{aligned}
$$

and $E_{1} \leq E_{2} \leq E_{3} \leq E_{4}$ are the edges of the valence bands. ${ }^{5}$ For more details on the function $M(z)$ we refer to Refs. [22,25] since we will limit ourselves to real order parameters $M(z)$ in the following.

For real order parameters $M(z)$ we have $\rho(\lambda)=\rho(-\lambda)$ or equivalently $E_{1}=-E_{4}, E_{2}=-E_{3}$. So there are only two parameters to specify the solution, which we choose to

\footnotetext{
${ }^{5}$ The expression for $\left\langle|M(z)|^{2}\right\rangle$ differs from that in Ref. [25] in that everything is expressed through the edges of the valence bands $\left\{E_{i}\right\}$. Since we will limit ourself to real condensates below, we do not give the tedious derivation. Alternatively, for a specifically given $M(z)$ it can be directly computed from the order parameter.
} 
be given through $E_{3}=\sqrt{1-\nu} \Delta$ and $E_{4}=\Delta$ [18]. This case is of particular interest since it is possible to generalize the solution to finite current quark masses [19]. The order parameter then has the specific form

$$
\begin{aligned}
M(z)= & \Delta(\nu \operatorname{sn}(b \mid \nu) \operatorname{sn}(\Delta z \mid \nu) \operatorname{sn}(\Delta z+b \mid \nu) \\
& \left.+\frac{\operatorname{cn}(b \mid \nu) \operatorname{dn}(b \mid \nu)}{\operatorname{sn}(b \mid \nu)}\right),
\end{aligned}
$$

where $\Delta$ is a scale parameter and sn, cn, dn are elliptic Jacobi functions with elliptic modulus $\sqrt{\nu}$, which physically describes lattices of equidistant solitions. Because of periodicity the parameters, $b$ and $\nu$ can be limited to $b \in$ $[0, \mathbf{K}(\nu)]$ and $\nu \in[0,1]$ with the quarter period $\mathbf{K}(\nu)$. The chiral limit, i.e. vanishing quark masses, corresponds to $b=\mathbf{K}(\nu)$.

In terms of the eigenvalue spectrum, the case of finite current quark masses included through $b \neq \mathbf{K}(\nu)$ only leads to a shift in the distribution of the eigenvalues $\lambda$

$$
\lambda \rightarrow \operatorname{sign}(\lambda) \sqrt{\lambda^{2}+\delta \Delta^{2}}, \quad \delta=\frac{1}{\operatorname{sn}^{2}(b \mid \nu)}-1 \geq 0
$$

and as a consequence the sum over the eigenvalue spectrum for arbitrary functions $f(\lambda)$ can be performed by using the density of states $\rho(\lambda)$ of the chiral limit

$$
\begin{aligned}
\frac{1}{V_{\|}} \sum_{\lambda} f(\lambda) & =\int_{-\infty}^{\infty} d \lambda \rho(\lambda) f(\lambda) \\
& \stackrel{\delta \neq 0}{\rightarrow} \int_{-\infty}^{\infty} d \lambda \rho(\lambda) f\left(\lambda \sqrt{1+\delta \Delta^{2} / \lambda^{2}}\right) .
\end{aligned}
$$

Addressing self-consistency and following Ref. [19], we have for the normalized eigenvectors $\psi_{1 D}(z)$ of $H_{1 D}$

$$
\begin{aligned}
\bar{\psi}_{1 D}(z) \psi_{1 D}(z)= & \frac{\lambda}{\lambda^{2}-\delta \Delta^{2}-\Delta^{2} \mathbf{E}(\nu) / \mathbf{K}(\nu)} M(z) \\
& -\frac{\Delta^{3} \sqrt{\delta(\delta+1)(\delta-\nu+1)}}{\lambda\left(\lambda^{2}-\delta \Delta^{2}-\Delta^{2} \mathbf{E}(\nu) / \mathbf{K}(\nu)\right)} .
\end{aligned}
$$

As discussed in the context of Eq. (21), we observe that the first term in Eq. (28) is proportional to the modulation $M(z)$, whereas the second is constant for each eigenvector. This enables us to obtain self-consistency in the gap equation for a proper choice of parameters. In particular, we find that the second term vanishes for $\delta=0$, which then corresponds to the chiral limit. Furthermore, we have

$$
\bar{\psi}_{\lambda, \mathbf{0}}\left(1-\gamma^{5}\right) \psi_{\lambda, \mathbf{0}}=\bar{\psi}_{1 D} \psi_{1 D}
$$

and conclude with the same reasoning that we can achieve self-consistency for $M(z)$ being a one-dimensional modulation in the NJL model.

\section{ONE-DIMENSIONAL MODULATIONS IN THE NJL MODEL}

We will now concentrate on real order parameters $M(z)$ and parametrize the band edges by $E_{3}=\sqrt{1-\nu} \Delta, E_{4}=$ $\Delta$, which corresponds to the order parameter in Eq. (25) for $b=\mathbf{K}(\nu)$. The reasons for discarding complex order parameters will be discussed in Sec. VI. After a nontrivial computation using Eq. (23) we obtain the density of states

$$
\rho(\lambda)= \begin{cases}\frac{1}{\pi} \frac{\lambda^{2}-\Delta^{2} \mathbf{E}(\nu) / \mathbf{K}(\nu)}{\sqrt{\left(\lambda^{2}-\Delta^{2}\right)\left(\lambda^{2}-(1-\nu) \Delta^{2}\right)}}, & \left(\lambda^{2}-\Delta^{2}\right)\left(\lambda^{2}-(1-\nu) \Delta^{2}\right)>0 \\ 0, & \text { else }\end{cases}
$$

which of course agrees with the finding in Ref. [18]. The evaluation of the thermodynamic potential in Eq. (11) then reduces to evaluating expressions of the form

$$
\begin{aligned}
\frac{1}{V_{\|}} \sum_{\lambda} \int \frac{d^{2} \mathbf{p}_{\perp}}{(2 \pi)^{2}} f\left(\lambda \sqrt{1+\mathbf{p}_{\perp}^{2} / \lambda^{2}}\right) & \rightarrow 2 \int_{-\infty}^{\infty} d \lambda \int \frac{d^{2} \mathbf{p}_{\perp}}{(2 \pi)^{2}} \rho(\lambda) f\left(\operatorname{sign}(\lambda) \sqrt{\lambda^{2}+\mathbf{p}_{\perp}^{2}+\delta \Delta^{2}}\right) \\
& =\int_{-\infty}^{\infty} d \lambda \int \frac{d^{2} \mathbf{p}_{\perp}}{(2 \pi)^{2}} \rho(\lambda) \tilde{f}\left(\sqrt{\lambda^{2}+\mathbf{p}_{\perp}^{2}+\delta \Delta^{2}}\right) \\
& =\frac{1}{2 \pi} \int_{0}^{\infty} d E E^{2} \int_{-1}^{1} d u \rho(E u) \tilde{f}\left(\sqrt{E^{2}+\delta \Delta^{2}}\right)=\int_{0}^{\infty} d E \tilde{\rho}(E) \tilde{f}\left(\sqrt{E^{2}+\delta \Delta^{2}}\right) .
\end{aligned}
$$

Here, we introduced $\tilde{f}(x)=f(x)+f(-x)$, used $\rho(\lambda)=\rho(-\lambda)$ and implicitly defined the effective density of states

$$
\tilde{\rho}(E)=\frac{1}{2 \pi} \int_{-1}^{1} d u E^{2} \rho(E u) .
$$

The overall factor of 2 in Eq. (31) stems from the degeneracy of the two Hamiltonians $H_{1 D}(M)$ and $H_{1 D}\left(M^{*}\right)$ in $H_{\mathrm{MF} ; 1 D}$. A straightforward but somewhat tedious calculation using the density of states in Eq. (30) then gives the explicit expression 


$$
\begin{aligned}
\tilde{\rho}(E)= & \theta(\sqrt{\tilde{\nu}} \Delta-E) \frac{\mathbf{E}(\tilde{\theta} \mid \tilde{\nu})+(\mathbf{E}(\nu) / \mathbf{K}(\nu)-1) \mathbf{F}(\tilde{\theta} \mid \tilde{\nu})}{\pi^{2}} E \Delta \\
& +\theta(E-\sqrt{\tilde{\nu}} \Delta) \theta(\Delta-E) \frac{\mathbf{E}(\tilde{\nu})+(\mathbf{E}(\nu) / \mathbf{K}(\nu)-1) \mathbf{K}(\tilde{\nu})}{\pi^{2}} E \Delta \\
& +\theta(E-\Delta) \frac{\mathbf{E}(\theta \mid \tilde{\nu})+\mathbf{E}(\nu) / \mathbf{K}(\nu) \mathbf{F}(\theta \mid \tilde{\nu})-\mathbf{F}(\theta \mid \tilde{\nu})+\sqrt{\left(E^{2}-\Delta^{2}\right)\left(E^{2}-\tilde{\nu} \Delta^{2}\right)} /(E \Delta)}{\pi^{2}} E \Delta,
\end{aligned}
$$

where we introduced the elliptic integrals of 1st and 2nd kind as well as $\tilde{\nu}=1-\nu, \tilde{\theta}=\arcsin (E /(\sqrt{\tilde{\nu}} \Delta))$ and $\theta=$ $\arcsin (\Delta / E)$. As an interesting cross-check we can consider the limit

$$
\left.\tilde{\rho}(E)\right|_{\nu=1}=\frac{1}{\pi^{2}} \theta(E-\Delta) \sqrt{\left(E^{2}-\Delta^{2}\right)} E,
$$

which is the effective density of states in an homogeneous phase with a quasiparticle gap $\Delta$ and

$$
\left.\tilde{\rho}(E)\right|_{\nu=0}=\frac{1}{\pi^{2}} E^{2},
$$

which corresponds to the ultrarelativistic gas. We also find for the asymptotic behavior of the effective density of states

$$
\begin{aligned}
\tilde{\rho}(E)= & \frac{E^{2}}{\pi^{2}}-\frac{\left\langle M(z)^{2}\right\rangle}{2 \pi^{2}}-\frac{\left\langle M(z)^{4}\right\rangle+\left\langle M^{\prime}(z)^{2}\right\rangle}{8 \pi^{2} E^{2}} \\
& +O\left(\left(\frac{1}{E}\right)^{4}\right) .
\end{aligned}
$$

For the considered one-dimensional modulation the thermodynamic potential in Eq. (11) can therefore be cast into

$$
\begin{aligned}
\Omega_{\mathrm{MF}, \mathrm{NJL}}(T, \mu ; \Delta, \nu, \delta)= & -2 N_{c} \int_{0}^{\infty} d E \tilde{\rho}(E) \\
& \times \tilde{f}_{\text {bare }}\left(\sqrt{E^{2}+\delta \Delta^{2}}\right) \\
& +\frac{1}{4 G_{s} L} \int_{0}^{L} d z|M(z)-m|^{2} \\
& + \text { const, }
\end{aligned}
$$

where $L$ is the period of the modulation and

$$
\begin{aligned}
\tilde{f}_{\text {bare }}(x)= & T \ln \left(2 \cosh \left(\frac{x-\mu}{2 T}\right)\right)+T \ln \left(2 \cosh \left(\frac{x+\mu}{2 T}\right)\right) \\
= & \tilde{f}_{\mathrm{UV}}(x)+\tilde{f}_{\text {medium }}(x), \\
\tilde{f}_{\mathrm{UV}}(x)= & x \\
\tilde{f}_{\text {medium }}(x)= & T \ln \left(1+\exp \left(-\frac{x-\mu}{T}\right)\right) \\
& +T \ln \left(1+\exp \left(-\frac{x+\mu}{T}\right)\right) .
\end{aligned}
$$

The last missing step is then a regularization of the diverging integration. For homogeneous phases this is mostly done by a momentum regularization (see e.g. Ref. [1]).
This is however not possible for inhomogeneous phases since the quasiparticle energies can no longer be labeled by a conserved three-momentum. Instead, we have to apply a regularization of the functional logarithm, e.g. by a propertime regularization, which is essentially a regularization acting on the energy spectrum instead of the quasiparticle momenta. For this purpose we have already identified the divergent vacuum contribution associated to $\tilde{f}_{\mathrm{UV}}(x)$, which we regularize by a specific blocking function in the propertime integral leading to a Pauli-Villars regularization of the form [26]

$$
\tilde{f}_{\mathrm{UV}}(x) \rightarrow \tilde{f}_{\mathrm{PV}}(x)=\sum_{j=0}^{3} c_{j} \sqrt{x^{2}+j \Lambda^{2}},
$$

with $c_{0}=1, c_{1}=-3, c_{2}=3, c_{3}=-1$, and a cutoff scale $\Lambda$.

With the expression of the thermodynamic potential as a function of $\Delta, \nu$, and $\delta$ we are now able to perform numerical investigations by performing the energy integration numerically and minimizing in the mentioned parameters. The resulting phase diagrams will of course depend on the choice of the model parameters $G_{s}, \Lambda$ and $m$. Those can be related to the chiral condensate $\langle\bar{\psi} \psi\rangle$ and the piondecay constant though the expressions [26]

$$
\begin{aligned}
\langle\bar{\psi} \psi\rangle & =-\frac{3 M_{q}}{4 \pi^{2}} \sum_{j=0}^{3} c_{j}\left(M_{q}^{2}+j \Lambda^{2}\right) \log \left(\frac{M_{q}^{2}+j \Lambda^{2}}{M_{q}^{2}}\right), \\
f_{\pi}^{2} & =-\frac{N_{c} M_{q}^{2}}{4 \pi^{2}} \sum_{j=0}^{3} c_{j} \log \left(\frac{M_{q}^{2}+j \Lambda^{2}}{M_{q}^{2}}\right),
\end{aligned}
$$

where $M_{q}$ is the constituent mass for the chirally broken phase in the vacuum.

\section{A NOTE ON PSEUDOSCALAR CONDENSATES}

Having worked out an expression for the thermodynamic potential for real order parameters $M(z)$, we may also discuss the more general case of a complex order parameter or in other words the relevance of pseudoscalar condensates $P_{a}(x)$.

On the one hand, this is less attractive from a technical point of view, since we only know self-consistent solutions away from the chiral limit for real order parameters $M(z)$. More importantly, we can also show that these phases are energetically less preferred, at least in the vicinity of a 
second order phase transition to the chirally restored phase and in the chiral limit. This has strong similarities to inhomogeneous phases in superconductors for which complex values of the order parameter are typically energetically disfavored $[27,28]$ and the fact that a plane wave is preferred in $1+1$ dimensions is misleading.
For this purpose we consider the generalized GinzburgLandau (GL) functional, which is a systematic expansion of the thermodynamic potential in the magnitude of the order parameter $M(z)$ as well as in gradients acting on it. Both are treated to be of the same order. For the chiral GN model this has been worked out in Ref. [29], where

$$
\begin{aligned}
\Omega_{\mathrm{GL}, \mathrm{GN}}(M)-\Omega_{\mathrm{GL}, \mathrm{GN}}(0)= & \frac{\alpha_{2}}{2}|M|^{2}+\frac{\alpha_{3}}{3} \operatorname{Im}\left(M M^{\prime *}\right)+\frac{\alpha_{4}}{4}\left(|M|^{4}+\left|M^{\prime}\right|^{2}\right)+\frac{\alpha_{5}}{5} \operatorname{Im}\left(\left(M^{\prime \prime}-3|M|^{2} M\right) M^{\prime *}\right) \\
& +\frac{\alpha_{6}}{6}\left(|M|^{6}+3|M|^{2}\left|M^{\prime}\right|^{2}+2|M|^{2}\left|M^{2}\right|^{\prime}+\frac{1}{2}\left|M^{\prime \prime}\right|^{2}\right)+\ldots
\end{aligned}
$$

has been derived. The dependence on temperature and chemical potential is hidden in the GL coefficients $\alpha_{i}$ and the details are irrelevant for the present discussion. For the NJL model the GL expansion for real order parameters has been worked out in Ref. [17] and a remarkable similarity to the GN model has been found when restricting to one-dimensional modulations. From the viewpoint of Lorentz symmetry as presented in Sec. III this may not be surprising since the GL coefficients are defined as integrals over functions in momentum space, so that at least for $\mathbf{p}_{\perp}=0$ the integrands of chiral GN and NJL model have to be equal up to an overall prefactor. Since the rotationally invariant extension to $\mathbf{p}_{\perp} \neq 0$ is unique, the structure of the GL expansions has to be the same.

In contrast to the chiral GN model the NJL Hamiltonian is however the direct product of $H_{1 D}(M)$ and $H_{1 D}\left(M^{*}\right)$. Therefore, the structure of the GL functional for the NJL model limited to one-dimensional modulations corresponds to the sum of that in Eq. (41) and its conjugate. We therefore obtain

$$
\begin{aligned}
\Omega_{\mathrm{GL}, \mathrm{NJL}}(M)-\Omega_{\mathrm{GL}, \mathrm{NJL}}(0)= & \frac{\beta_{2}}{2}|M|^{2}+\frac{\beta_{4}}{4}\left(|M|^{4}+\left|M^{\prime}\right|^{2}\right) \\
& +\frac{\beta_{6}}{6}\left(|M|^{6}+3|M|^{2}\left|M^{\prime}\right|^{2}\right. \\
& \left.+2|M|^{2}\left|M^{2}\right|^{\prime}+\frac{1}{2}\left|M^{\prime \prime}\right|^{2}\right) \\
& +\ldots,
\end{aligned}
$$

where $\beta_{i}$ are the GL coefficients of the NJL model as stated in Ref. [17].

With the generic form of the GL functional at hand, we can now compare various ansätze for $M(z)$. We will limit ourselves to second order phase transitions from an inhomogeneous to the chirally restored phase and therefore to a regime where $\beta_{2}>0, \beta_{4}<0$. Furthermore all Fourier modes of $M(z)$ decouple to order $M^{2}$ due to momentum conservation in the GL coefficients, which are calculated in an homogeneous background. For this reason we consider only the two extremes

$$
M_{F F}(z)=\frac{\Delta_{F F}}{\sqrt{2}} \exp (i q z), \quad M_{\sin }(z)=\Delta_{\sin } \sin (q z) .
$$

For an individually optimized wave vector $q$ the thermodynamic potentials for these ansätze is then given by

$$
\begin{aligned}
\Omega_{\mathrm{GL}, \mathrm{NJL}}\left(M_{F F}\right)-\Omega_{\mathrm{GL}, \mathrm{NJL}}(0)= & \left(\frac{\beta_{2}}{4}-\frac{3 \beta_{4}^{2}}{32 \beta_{6}}\right) \Delta_{F F}^{2} \\
& -\frac{1}{8} \beta_{4} \Delta_{F F}^{4}+O\left(\Delta_{F F}^{5}\right), \\
\Omega_{\mathrm{GL}, \mathrm{NJL}}\left(M_{\mathrm{sin}}\right)-\Omega_{\mathrm{GL}, \mathrm{NJL}}(0)= & \left(\frac{\beta_{2}}{4}-\frac{3 \beta_{4}^{2}}{32 \beta_{6}}\right) \Delta_{\text {sin }}^{2} \\
& -\frac{1}{16} \beta_{4} \Delta_{\text {sin }}^{4}+O\left(\Delta_{\mathrm{sin}}^{5}\right) .
\end{aligned}
$$

From this result we can deduce that the phase transition for both cases happens at the same values of the GL coefficients, namely, at $\beta_{2}-\frac{3 \beta_{4}^{2}}{8 \beta_{6}}=0$. The inhomogeneous phase then occurs for $\beta_{2}-\frac{3 \beta_{4}^{2}}{8 \beta_{6}}<0$, where the coefficient of the $\Delta_{F F}^{2}$ and $\Delta_{\text {sin }}^{2}$ term is negative. Since the coefficient of the $\Delta_{\text {sin }}^{4}$ term is however smaller than that of the $\Delta_{F F}^{4}$ term (recall that $\beta_{4}<0$ ), we find that the modulation of the form $M_{\sin }(z)$ is energetically preferred compared to $M_{F F}(z)$ for optimized parameters. In this sense, the ground state with a real order parameters wins over the plane wave. Two brief comments are in order here: First, the self-consistent modulations discussed in the previous section indeed become sinusoidal when approaching the phase transition to the chirally restored phase and second, the sinusoidal modulation can of course be understood as two plane waves in opposite directions. The latter have also been found energetically preferred compared to the plane wave in inhomogeneous (color-)superconductors [27,28].

Beyond the GL expansion, we can also directly compare the mean-field results for soliton lattice and for the dual chiral density wave. Following the idea of using the knowledge obtained within $1+1$ dimensional models, we start with the density of states $[22]^{6}$

\footnotetext{
${ }^{6}$ The mass function here is parametrized as $M(z)=$ $\Delta \exp (2 i q z)$. The density of states can also be obtained from Eq. (23) using $E_{1}=E_{2}=E_{3}=\Delta-q$ and $E_{4}=\Delta+q$.
} 


$$
\rho_{\mathrm{CDW}}(\lambda)=\frac{1}{\pi} \frac{|\lambda-q|}{\sqrt{(\lambda-q)^{2}-\Delta^{2}}} \theta\left((\lambda-q)^{2}-\Delta^{2}\right),
$$

use Eq. (32) and arrive at the effective density of states

$$
\begin{aligned}
\tilde{\rho}_{\mathrm{CDW}}(E)= & \frac{E \sqrt{(E-q)^{2}-\Delta^{2}}}{2 \pi^{2}} \theta(E-q-\Delta) \\
& +\frac{E \sqrt{(E+q)^{2}-\Delta^{2}}}{2 \pi^{2}} \theta(E-q+\Delta) \theta(E+q-\Delta) \\
& +\frac{E\left(\sqrt{(E+q)^{2}-\Delta^{2}}-\sqrt{(E-q)^{2}-\Delta^{2}}\right)}{2 \pi^{2}} \\
& \times \theta(q-\Delta-E),
\end{aligned}
$$

where we assumed the convention $q>0, \Delta>0$. The thermodynamic potential is therefore

$$
\begin{aligned}
\Omega_{\mathrm{MF}, \mathrm{NJL} ; \mathrm{CDW}}(T, \mu ; \Delta, q)= & -2 N_{c} \int_{0}^{\infty} d E \tilde{\rho}_{\mathrm{CDW}}(E) \\
& \times\left(\tilde{f}_{\mathrm{PV}}(E)+\tilde{f}_{\mathrm{med}}(E)\right) \\
& +\frac{\Delta^{2}}{4 G_{s}}+\text { const },
\end{aligned}
$$

which can be evaluated and minimized numerically. Numerical investigations then confirm the expectations from the GL analysis.

\section{QM MODEL AND MEAN-FIELD APPROXIMATION}

The NJL model regularized by a proper-time regularization and adjusted to chiral condensate and pion-decay constant is known to give constituent quark masses of order $200 \mathrm{MeV}$ in the vacuum. Hence, it gives an undesired phenomenology with regard to the QCD phase diagram, mainly because quasiparticles will start forming a Fermi surface at $\mu \simeq M_{q}$. This is phenomenologically unacceptable for $\mu<\left(M_{N}-B\right) / 3 \simeq 308 \mathrm{MeV}$, where $M_{N}$ is the nucleon mass and $B$ the binding energy of nucleons in nuclear matter. In contrast, the NJL model regularized by a sharp three-momentum cutoff gives constituent quark masses in the vacuum significantly larger than $300 \mathrm{MeV}$. Since we have not found a regularization that avoids these problems, we instead also introduce a model that is very similar to the NJL model and where the issue of the regularization scheme can be surpassed: The linear sigma model, which in this context is usually named the QM model [30,31].

The Lagrangian of the QM model with $N_{f}=2$ and $N_{c}=3$ is given by

$$
\mathcal{L}_{\mathrm{QM}}=\bar{\psi}\left(i \gamma^{\mu} \partial_{\mu}-g\left(\sigma+i \gamma_{5} \tau^{a} \pi^{a}\right)\right) \psi-U\left(\sigma, \pi^{a}\right),
$$

where

$$
\begin{aligned}
U\left(\sigma, \pi^{a}\right)= & -\frac{1}{2}\left(\partial_{\mu} \sigma \partial^{\mu} \sigma+\partial_{\mu} \pi^{a} \partial^{\mu} \pi^{a}\right) \\
& +\frac{\lambda}{4}\left(\sigma^{2}+\pi^{a} \pi^{a}-v^{2}\right)^{2}-c \sigma,
\end{aligned}
$$

$\psi$ is again the $4 N_{f} N_{c}$-dimensional quark spinor, $\sigma$ the scalar field of the $\sigma$ meson and $\pi^{a}$ the pseudoscalar fields of the pion triplet. In mean-field approximation we treat the fields $\sigma$ and $\pi^{a}$ as classical and replace them by there expectation values [30,31]. Furthermore, we can use lowenergy relations to connect the parameters $c, g, \lambda$, and $v^{2}$ with hadronic observables. We will express those by the pion-decay constant $f_{\pi}$, the constituent quark mass in the vacuum $M_{q}$, the pion mass $m_{\pi}$ and $\sigma$-meson mass $m_{\sigma}$ via $\langle\sigma\rangle=f_{\pi},\left\langle\pi^{a}\right\rangle=0, c=m_{\pi}^{2} f_{\pi}, g=M_{q} / f_{\pi}, \lambda=\left(m_{\sigma}^{2}-\right.$ $\left.m_{\pi}^{2}\right)^{2} /\left(2 f_{\pi}^{2}\right)$ and $v^{2}=f_{\pi}^{2}-m_{\pi}^{2} / \lambda$.

For the thermodynamic potential in mean-field approximation we only include the contributions of the fermionic fluctuations and approximate

$$
\begin{aligned}
\Omega_{\mathrm{QM}}\left(T, \mu ; \sigma(\mathbf{x}), \pi^{a}(\mathbf{x})\right) & =-\frac{T}{V} \ln \int \mathcal{D} \bar{\psi} \mathcal{D} \psi \mathcal{D} \sigma \mathcal{D} \pi^{a} \exp \left(\int_{x \in[0,(1 / T)] \times V}\left(\mathcal{L}_{\mathrm{QM}}+\mu \bar{\psi} \gamma^{0} \psi\right)\right) \\
& \stackrel{\text { mean-field }}{\rightarrow}-\frac{T N_{c}}{V} \sum_{n} \operatorname{Tr}_{D, f, V} \log \left(\frac{1}{T}\left(i \omega_{n}+\tilde{H}_{\mathrm{MF}, \mathrm{QM}}-\mu\right)\right)+\frac{1}{V} \int_{V} U\left(\sigma(\mathbf{x}), \pi^{a}(\mathbf{x})\right),
\end{aligned}
$$

where $\sigma(\mathbf{x})$ and $\pi^{a}(\mathbf{x})$ are nonvanishing expectation values of the respective fields. Limiting to cases with $\pi^{1}(\mathbf{x})=$ $\pi^{2}(\mathbf{x})=0$ the Hamiltonian reads

$$
\tilde{H}_{\mathrm{MF}, \mathrm{QM}}=-i \gamma^{0} \gamma^{i} \partial_{i}+\gamma^{0}\left(g \sigma(\mathbf{x})+i g \gamma^{5} \tau^{3} \pi^{3}(\mathbf{x})\right),
$$

and we have to evaluate the same functional tracelogarithm as in the case of the NJL model, but now with the identification $M(\mathbf{x})=g\left(\sigma(\mathbf{x})+i \pi^{3}(\mathbf{x})\right)$.
The QM model is renormalizable, which means that the divergences in the functional trace logarithm can be absorbed by the model parameters. For this purpose it is useful to observe that we can always separate zero-point fluctuations from thermal fluctuations as indicated by $\tilde{f}_{\mathrm{UV}}(x)$ and $\tilde{f}_{\text {medium }}(x)$ in Eq. (38), respectively. Instead of a proper renormalization we will however follow Refs. [30,31], where it has been assumed that the contribution from zero-point approximations can well be approximated by $\frac{1}{V} \int_{V} U\left(\sigma(\mathbf{x}), \pi^{a}(\mathbf{x})\right)$ with the parameters 
directly adopted to phenomenology. As a result we can evaluate the thermodynamic potential for one-dimensional modulations $\sigma(\mathbf{x})=M(z) / g$ and $\pi^{a}(\mathbf{x})=0$ with $M(z)$ given in Eq. (25) on the same level as for the NJL model, and we get

$$
\begin{aligned}
\Omega_{\mathrm{MF}, \mathrm{QM}}(T, \mu ; \Delta, \nu, \delta) \\
\equiv \Omega_{\mathrm{MF}, \mathrm{QM}}(T, \mu ; M(z) / g, 0) \\
=-2 N_{c} \int_{0}^{\infty} d E \tilde{\rho}(E) \tilde{f}_{\text {medium }}\left(\sqrt{E^{2}+\delta \Delta^{2}}\right) \\
\quad+\frac{1}{L} \int_{0}^{L} d z U(M(z) / g, 0)+\text { const. }
\end{aligned}
$$

For completeness we have to denote that this will only be a self-consistent solution if $M(z)$ would solve the equations of motions associated to minimizing the functional $U\left(\sigma(\mathbf{x}), \pi^{a}(\mathbf{x})\right)$, which is in general not the case. This is not considered a major issue within this work since we already limited the space of possible solutions to that of one-dimensional modulations so that we cannot guarantee to find the true ground state anyway. Instead, we will find domains where at least one inhomogeneous phase is energetically preferred over all homogeneous phases and therefore pointing out domains where the ground state is for sure inhomogeneous (in the presented approximation).

At the end of this section we would like to point out that we can easily analyze the vicinity of the chiral critical point by a generalized Ginzburg-Landau approximation. For this purpose we first observe that the functional trace-logarithm in Eq. (50) is formally identical to the one in Eq. (3). The GL expansion of the latter has been worked out in Ref. [17], and the structure of the GL functional stays the same when limiting to thermal fluctuations. ${ }^{7}$ The GL expansion of $\Omega_{\mathrm{MF}, \mathrm{QM}}(T, \mu ; \sigma(\mathbf{x})=M(\mathbf{x}) / g, 0)$ can therefore easily deduced from Ref. [17]. Without giving unnecessary details for the presented discussion, the GL expansion with coefficients $\beta_{i}^{\prime}$ takes the form

$$
\begin{aligned}
\Omega_{\mathrm{MF}, \mathrm{QM}}(T, \mu ; \sigma(\mathbf{x})= & M(\mathbf{x}) / g, 0) \\
= & \frac{1}{V} \int_{V}\left(\frac{\beta_{2}^{\prime}}{2} M(\mathbf{x})^{2}+\frac{\beta_{4}^{\prime}}{4}\left(M(\mathbf{x})^{4}\right.\right. \\
& \left.+(\nabla M(\mathbf{x}))^{2}\right)+\frac{\beta_{6}^{\prime}}{6}\left(M(\mathbf{x})^{6}\right. \\
& \left.+5 M(\mathbf{x})^{2}(\nabla M(\mathbf{x}))^{2}+(\Delta M(\mathbf{x}))^{2}\right) \\
& +\frac{v^{4} \lambda}{4}-\operatorname{cg} M(\mathbf{x})-\frac{g^{2} v^{2} \lambda}{2} M(\mathbf{x})^{2} \\
& \left.+\frac{1}{4}\left(g^{2} \lambda M(\mathbf{x})^{4}+2 g^{2}(\nabla M(\mathbf{x}))^{2}\right)\right) \\
& +\ldots
\end{aligned}
$$

\footnotetext{
${ }^{7}$ The GL coefficients are defined through integrals in momentum space for which we can separate vacuum and thermal contributions.
}

Restricting to the chiral limit for simplicity, i.e. $c=0$, we can identify the chiral critical point as the position where the coefficients of the $M(\mathbf{x})^{2}$ term and the $M(\mathbf{x})^{4}$ term vanish, i.e.

$$
\beta_{2}^{\prime}-g^{2} v^{2} \lambda=0, \quad \beta_{4}^{\prime}+g^{2} \lambda=0 .
$$

Considering the coefficient of the $(\nabla M(\mathbf{x}))^{2}$ term at this point we find

$$
\frac{\beta_{4}^{\prime}+2 g^{2}}{4}=\frac{g^{2}(2-\lambda)}{4},
$$

being negative for $\lambda>2$. Since homogeneous phases are instable for a vanishing $M(\mathbf{x})^{2}$ term and a negative $(\nabla M(\mathbf{x}))^{2}$ term, the chiral critical point would be hidden in this case since an inhomogeneous phase was energetically preferred at its location. This scenario is actually realized for phenomenological parameters since we have $\lambda=m_{\sigma}^{2} /\left(2 f_{\pi}^{2}\right) \sim 20$. Because of the involved approximations we do however not want to overemphasize this result.

\section{NUMERICAL RESULTS}

With the expressions for the thermodynamic potentials of the NJL model and the QM model in Eq. (37) [combined with the described regularization] and Eq. (52) at hand, we can now study the structure of the phase diagram numerically. Except for the analytically known averages of $M(z)$, $M(z)^{2}$, and $M^{\prime}(z)^{2}$ given in Ref. [19], we evaluate all involved integrals numerically and obtain $\Omega_{\mathrm{MF}, \mathrm{NJL} / \mathrm{QM}}(T, \mu ; \Delta, \nu, \delta)$. Since numerical minimizations typically determine parameters up to a total and/or relative error and since elliptic integrals depend on the elliptic modulus $\nu$ in a highly nonlinear fashion for $\nu \rightarrow 1$, it is numerically very helpful to express $\nu=1-$ $16 \exp \left(-4 \ln (2) / q_{h}\right)$ with $q_{h} \in[0,1]$ and then minimize in the parameters $\Delta, q_{h}$, and $\delta$.

We will now start discussing results for the NJL phase diagram in the chiral limit, i.e. for $m=0$. For the reasons discussed at the beginning of Sec. VII, we will fix $f_{\pi}=$ $88 \mathrm{MeV}$ to its value in the chiral limit and instead of adjusting $\langle\bar{\psi} \psi\rangle$, we choose a value for $M_{q}$. These choices in turn fix the model parameters through Eq. (40). On the left side of Fig. 1 we present the phase diagram restricting to homogeneous phases for a value of $M_{q}=300 \mathrm{MeV}$. For larger temperatures we see the second order phase transition line from the chirally broken to the restored phase, which turns into a first order phase transition line at ( $\mu_{\mathrm{cr}}=$ $269 \mathrm{MeV}, T_{\text {cr }}=74 \mathrm{MeV}$ ) locating the chiral critical point. The first order line then ends at $(\mu=312 \mathrm{MeV}$, $T=0 \mathrm{MeV}$ ), where the associated spinodal region spannes about $18 \mathrm{MeV}$ in the quark chemical potential $\mu_{q}$. Although the chiral condensate with $\langle\bar{\psi} \psi\rangle=$ $-(193 \mathrm{MeV})^{3}$ for this case is phenomenologically too small, we observe that the structure of the phase diagram 

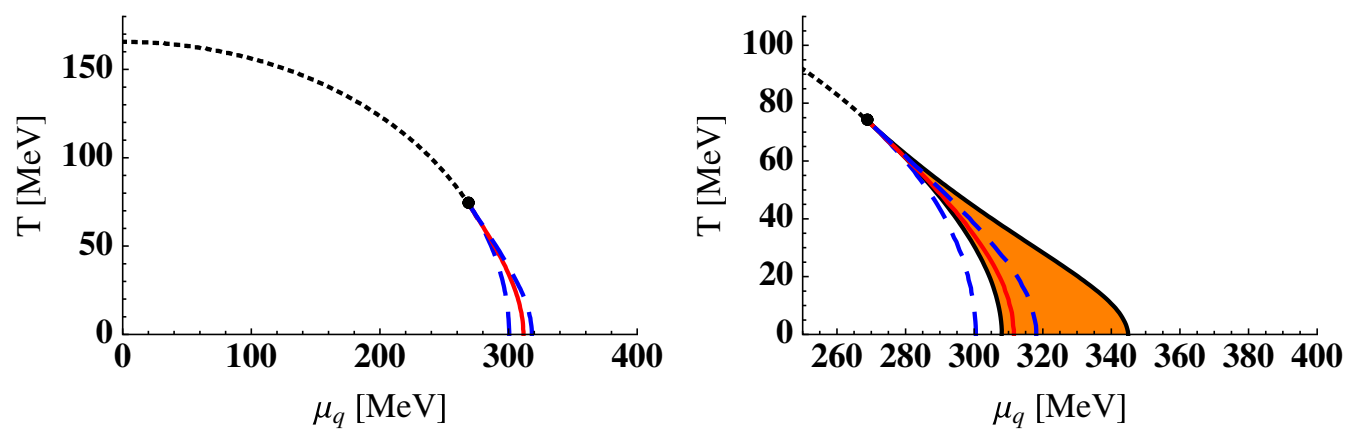

FIG. 1 (color online). Left: Structure of the NJL phase diagram in the chiral limit as a function of temperature $T$ and quark chemical potential $\mu_{q}$ for $M_{q}=300 \mathrm{MeV}$. The black (short-dashed) line indicates the second order phase transition from the chirally broken to restored phase, the red (solid) line the first order phase transition and the bullet the critical point. The spinodal region is enclosed by the blue (long-dashed) lines. Right: Same plot as on the left including the orange (shaded) domain where the energetically preferred ground state is inhomogeneous.

is similar to that typically found in NJL models using a sharp three-momentum cutoff [30].

Focusing on the region near/around the first order phase transition and the critical point, the right-hand side of Fig. 1 shows the same lines as on the left, but now also including the domain where inhomogeneous phases are energetically preferred. As discussed in Ref. [17] for the vicinity of the critical point, we observe that there is no longer a first order phase transition in the phase diagram, since it is replaced by an inhomogeneous ground state. The transitions from the chirally broken to the inhomogeneous and from the inhomogeneous to the restored phase are both second order, where the first transition is characterized by the formation of (in the perpendicular direction) localized domain-wall solitons and the second by the melting of the condensate.

The nature of the phase transitions is also apparent in the squared spatial average $\left\langle M(x)^{2}\right\rangle^{1 / 2}$ of the order parameter as well as the wave vector of the one-dimensional modulation $q$. For vanishing temperatures these are depicted on the left of Fig. 2 for $M_{q}=250,300,350 \mathrm{MeV}$. We observe that $q$ continuously raises from $q=0$ at the transition from the chirally broken to the inhomogeneous phase, which is related to the formation of localized objects. On the other hand, $\left\langle M(x)^{2}\right\rangle^{1 / 2}$ continuously goes to zero at the transition from the inhomogeneous to the chirally restored phase. In the same plot we also see that at vanishing temperatures the constituent quark mass stays constant for $\mu_{q} \leq M_{q}$ (below any phase transition) and starts decreasing with the condensation of quarks.

Whether or not quarks condense before reaching a phase transition has tremendous consequences on the structure of the phase diagram as shown on the right-hand side of Fig. 2. Here, we present the region of the first order phase transition for $M_{q}=250,300,350 \mathrm{MeV}$. Qualitatively the picture for those three cases is similar, but quantitatively there are significant differences. Since for $M_{q}=250 \mathrm{MeV}$ the quarks already form a Fermi surface before reaching the phase transition, the order parameter is already diminished at the phase transition. As a result the phase transition is very weak and the spinodal region/domain of inhomogeneous phases strongly reduced. For $M_{q}=350 \mathrm{MeV}$ on the other side the interaction is very strong and there is no condensation before reaching the onset of inhomogeneous phase (or the first order phase transition when limiting to homogeneous phases). The domain of inhomogeneous
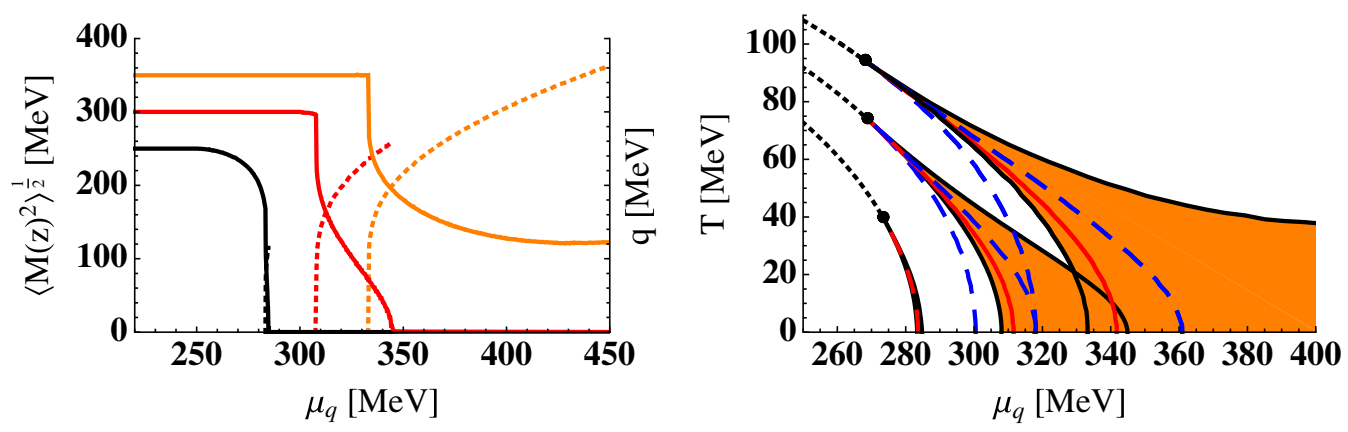

FIG. 2 (color online). Left: Wave vector $q$ (dashed lines) and average of constituent mass $\sqrt{\left\langle M(z)^{2}\right\rangle}$ (solid lines) at vanishing temperatures as function of quark chemical potential $\mu_{q}$ for $M_{q}=250 \mathrm{MeV}$ (black lines), $M_{q}=300 \mathrm{MeV}$ (red, dark grey lines lines) and $M_{q}=350 \mathrm{MeV}$ (orange, light grey lines lines). Right: Same plot as on the right of Fig. 1, now including results for $M_{q}=$ $350 \mathrm{MeV}$ (upper branch) and $M_{q}=250 \mathrm{MeV}$ (lower branch). 

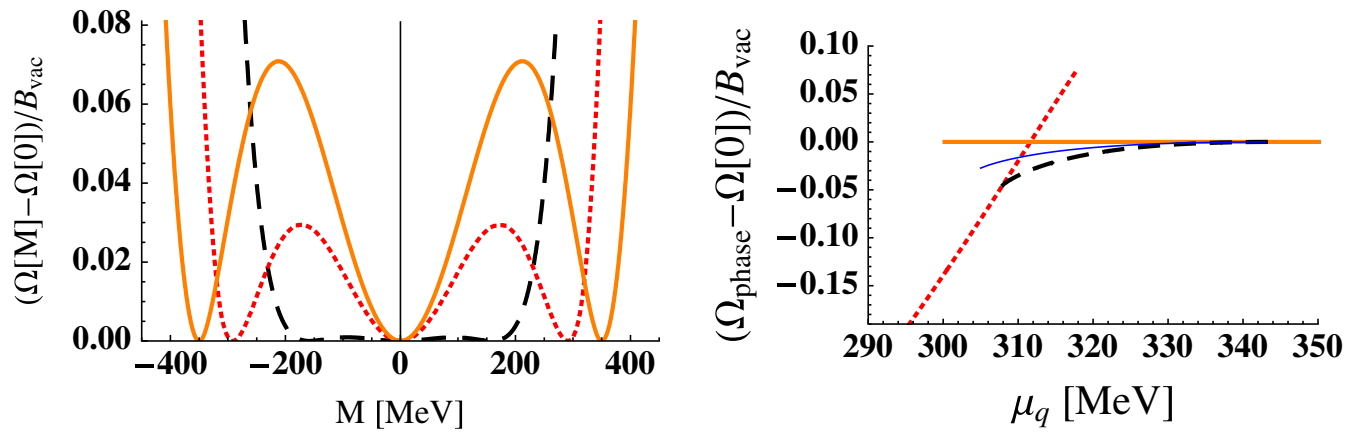

FIG. 3 (color online). Left: Difference of thermodynamic potential $\Omega(T, \mu ; M)$ in the chiral limit for the homogeneous phase with constituent mass $M$ and its value for $M=0$ in units of the bag constant $B_{\mathrm{vac}}$. The black (long-dashed) line corresponds to $M_{q}=$ $250 \mathrm{MeV}$, the red (short-dashed) to $M_{q}=300 \mathrm{MeV}$ and the orange (solid) to $M_{q}=350 \mathrm{MeV}$. All for vanishing temperatures and $\mu_{q}$ at the first order phase transition (when limiting to homogeneous phases). Right: Difference of thermodynamic potential $\Omega_{\text {phase }}$ in the

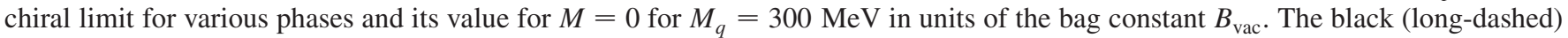
line corresponds to the inhomogeneous, the red (short-dashed) to the chirally broken and the orange (solid) to the chirally restored phase. In addition, also the corresponding result for the chiral density wave is shown as the blue (thin) line, never being energetically favored.

phases is very large here, and it is conceivable that its persistence beyond $\mu_{q}=400 \mathrm{MeV}$ is a regularization artefact. We also point out that although the value of constituent quark mass $M_{q}$ is comparable to that in NJL models using a three-momentum cutoff, the chiral condensate with $\langle\vec{\psi} \psi\rangle=-(186 \mathrm{MeV})^{3}$ at $M_{q}=350 \mathrm{MeV}$ decreases with increasing $M_{q}$ here.

The results indicate that the size of the domain for inhomogeneous ground states, similar to the spinodal region, is related to the strength of the first order phase transition. In order to give a better picture for the latter, the thermodynamic potentials as effective actions in the order parameter are shown on the left of Fig. 3 at vanishing temperatures and the respective first order phase transition points. We find that the local maxima between $M_{q}=$ $250 \mathrm{MeV}$ and $M_{q}=350 \mathrm{MeV}$ in the potential even when measured in the respective bag constants $B_{\text {vac }}=$ $\Omega(0,0 ; 0)-\Omega\left(0,0 ; M_{q}\right)$ differs by more than an order of magnitude, therefore underlining the quantitative differ- ence between the two cases. For completeness, the value of the bag constant for $M_{q}=250,300,350 \mathrm{MeV}$ is $B_{\mathrm{vac}}=$ $40,62,91 \mathrm{MeV} / \mathrm{fm}^{3}$, respectively.

Coming back to $M_{q}=300 \mathrm{MeV}$ we also like to give an impression of the thermodynamic potential at vanishing temperatures using the right-hand side of Fig. 3. Here, we show the value of the thermodynamic potential for the energetically most preferred homogeneous phase as well as the inhomogeneous phase. Plotted are only cases that form a local minimum of the thermodynamic potential as an effective action, such that, e.g. the spinodal region can be deduced from the plot. In the case of several local minima at a given $\mu_{q}$ the global minimum is energetically preferred, and we see that if existent, the inhomogeneous phase forms the ground state. In addition, the thermodynamic potential of the chiral density wave is included to underline that it is never energetically preferred. In agreement with the GL expansion discussed in Sec. VI, its pressure difference to the unbroken phase is about half of
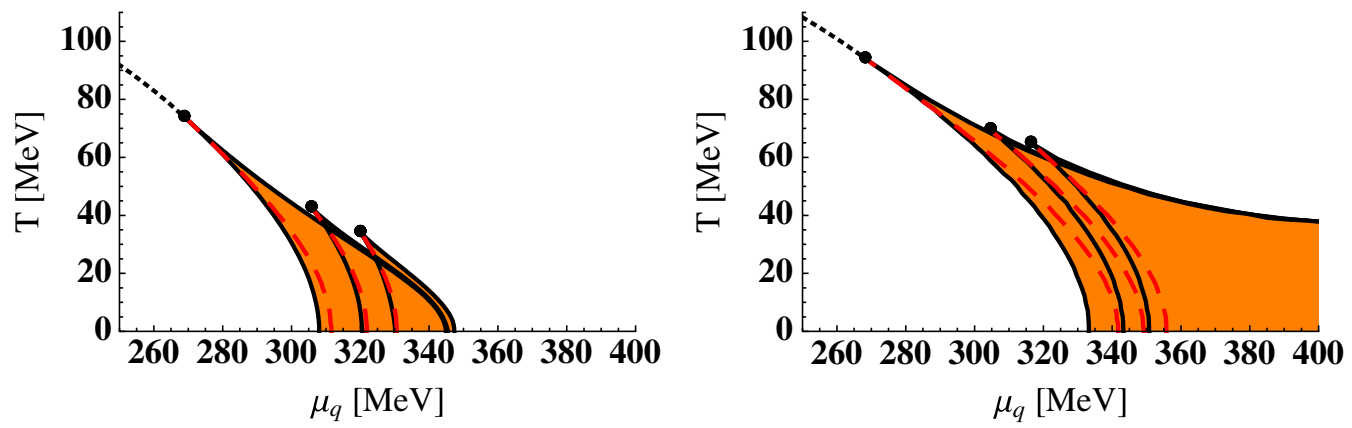

FIG. 4 (color online). Left: Same plot as on the right of Fig. 1, beside $m=0 \mathrm{MeV}$ now also including the domain of inhomogeneous phases for $m=5 \mathrm{MeV}$ and $m=10 \mathrm{MeV}$ (orange shaded domains). Branches with critical points (black bullets) at smaller temperature $T$ and larger quark chemical potential $\mu_{q}$ correspond to larger current quark masses. Right: Same plot as on the left for $M_{q}=350 \mathrm{MeV}$. 

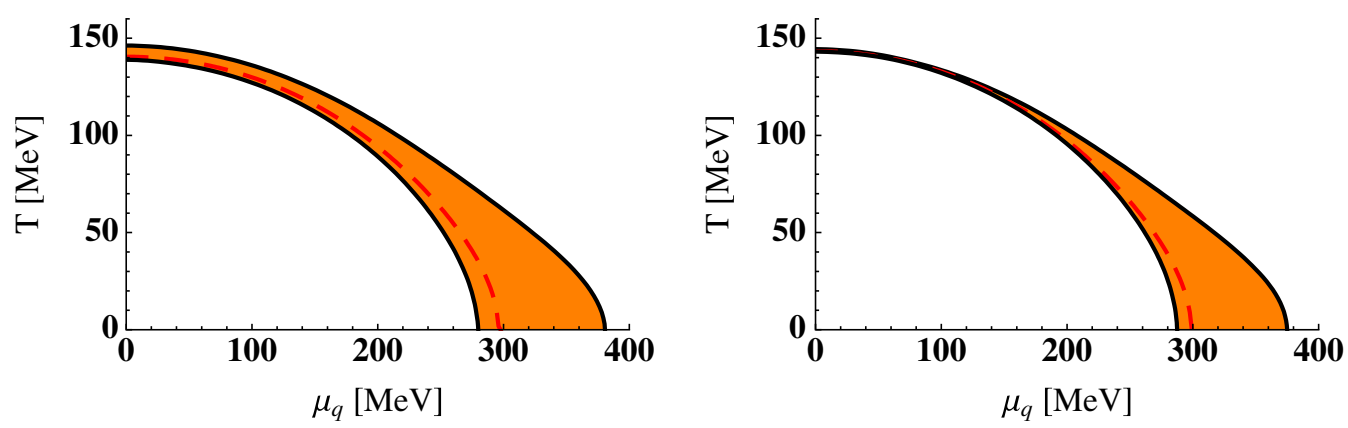

FIG. 5 (color online). Left: Phase diagram for the QM model in the chiral limit with the red (dashed) line indicating the first order phase transition when limiting to homogeneous phases only. Inhomogeneous phases are preferred in the orange (shaded) domain enclosed by black (solid) lines. Right: Same plot as on the left, now for $m_{\pi}=69 \mathrm{MeV}$.

that of the soliton lattice. Also, the order of the phase transitions can be deduced from the plot.

As a next point we want to address the case of finite current quark masses. For this purpose we again fix the parameters $G_{s}, \Lambda$ by choosing $f_{\pi}, M_{q}$ in the chiral limit and then turn on a finite current quark mass $m$. In Fig. 4, we present our results for the relevant part of the phase diagram choosing $m=0,5,10 \mathrm{MeV}$. Aside from the fact that the second order phase transition is turned into a crossover when leaving the chiral limit, we see that the qualitative picture stays the same: Again the first order phase transition line is replaced by two second order phase transition lines, which enclose a domain where inhomogeneous phases are energetically preferred. All lines meet at the critical point, which can be calculated on the bases of homogeneous phases only and which is known to shift toward smaller temperatures and larger quark chemical potentials when increasing $m$.

At the end of this section we sketch some results for the QM model and due to the similarity to the NJL model we will restrict to the structure of the phase diagram only. Following Refs. [30,31] we choose the model parameters by $f_{\pi}=93 \mathrm{MeV}, M_{q}=300 \mathrm{MeV}, m_{\sigma}=600 \mathrm{MeV}$ and vary the pion mass $m_{\pi}$. In Figs. 5 and 6 we show the phase diagrams including the domain for inhomogeneous phases with $m_{\pi}=0,69,138 \mathrm{MeV}$. The model in the applied

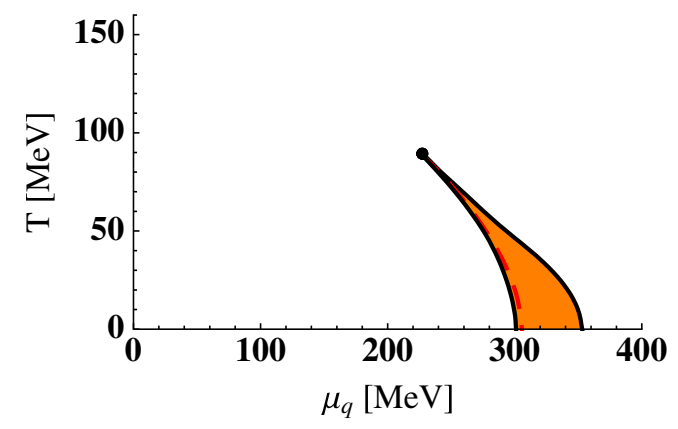

FIG. 6 (color online). Same plot as on the left of Fig. 5, now for $m_{\pi}=138 \mathrm{MeV}$ and with the bullet showing the critical point. approximation does not have a critical point in the chiral limit and as expected after the discussion of the model in Sec. VII and the numerical results for the NJL model, the first order phase transition from the chirally broken to the (partially) restored phase is replaced by two second order phase transitions at the boundary of a inhomogeneous domain. For half the physical pion mass $m_{\pi}=69 \mathrm{MeV}$ we find the same scenario, whereas we find a critical point in the phase diagram at the physical value of the pion mass $m_{\pi}=138 \mathrm{MeV}$. Although the critical point is in principle hidden by inhomogeneous phases as discussed at the end of Sec. VII, the Lifshitz point at the cusp of the two second order phase transition lines and the critical point appear to be so close that we cannot distinguish those numerically.

\section{DISCUSSION}

In this paper we have shown how inhomogeneous phases in the NJL model and the QM model with lowerdimensional modulations can be investigated and especially how this analysis is related that in the (chiral) Gross-Neveu model. As suggested in Ref. [17] we have confirmed that the first order phase transition line in the NJL phase diagram of homogeneous phases is completely replaced by two second order phase transition lines that border an inhomogeneous phase. The intersection of these lines defines a Lifshitz point, which coincides with the critical point also for finite current quark masses. For the QM model in the applied approximation this picture is somewhat modified as the critical point (in the case of homogeneous phases) is slightly hidden by an inhomogeneous phase. Although the qualitative behavior is very general, the specific structure of the phase diagram and, in particular, the importance of inhomogeneous phases is strongly depending on the model parameters. We have established that there is a close connection between the significance of inhomogeneous phases and the strength of the first order phase transition when limiting to homogeneous phases, in particular, the size of its spinodal region. In addition, the relevance of pseudoscalar condensates has been addressed. 
This work is a further step in the investigation of inhomogeneous phases within NJL-type models. In the following various related problems could be addressed: From a phenomenological point of view it would be interesting to study the interplay and competition with colorsuperconducting phases, being expected in the same domain of the phase diagram [1,2]. Also, the calculation of higher-dimensional modulations should be in the range of numerical approaches, especially since the magnitude of the order parameter and the size of the Brillouin zone can be large and a numerical determination of the density of states would therefore be possible [32]. More toward an extension of the considered models, a GL analyses including, e.g. vector-vector and 't Hooft interaction could be performed to check whether and how the relation of Lifshitz and chiral critical point can be modified. Also, inhomogeneous phases in the Polyakov-NJL model can be studied easily since the density of states for quasiparticles is not altered by the inclusion of the Polyakov loop. As a consequence, the technical issues associated with inhomo- geneous phases are not further complicated here. From a technical point of view, e.g. the zero-point energies in the QM model can be easily taken into account since the asymptotic behavior of the spectral density is known analytically. This would then allow for a proper renormalization of the model. In general it would of course also be interesting to study these phases beyond mean-field approximation, especially since fluctuations play a key role at a critical point as, e.g. discussed for the QM model in Ref. [33].

\section{ACKNOWLEDGMENTS}

We thank G. Basar, M. Buballa, S. Carignano, G. Dunne, K. Rajagopal, B.-J. Schaefer, and M. Stephanov for helpful comments and discussions. This work was supported in part by funds provided by the U.S. Department of Energy (D.O.E.) under Cooperative Research Agreement No. DEFG0205ER41360 and by the German Research Foundation (DFG) under Grant No. Ni 1191/1-1.
[1] K. Rajagopal and F. Wilczek, arXiv:hep-ph/0011333; T. Schäfer, arXiv:hep-ph/0304281; D. H. Rischke, Prog. Part. Nucl. Phys. 52, 197 (2004); M. Buballa, Phys. Rep. 407, 205 (2005).

[2] M. G. Alford, A. Schmitt, K. Rajagopal, and T. Schafer, Rev. Mod. Phys. 80, 1455 (2008).

[3] M. A. Stephanov, Proc. Sci. LAT2006 (2006) 024.

[4] K. Fukushima, Phys. Lett. B 591, 277 (2004).

[5] C. Ratti, M. A. Thaler, and W. Weise, Phys. Rev. D 73, 014019 (2006).

[6] B. J. Schaefer, J. M. Pawlowski, and J. Wambach, Phys. Rev. D 76, 074023 (2007).

[7] Y. Nambu and G. Jona-Lasinio, Phys. Rev. 122, 345 (1961).

[8] M. Gell-Mann and M. Levy, Nuovo Cimento 16, 705 (1960).

[9] D. V. Deryagin, D. Y. Grigoriev, and V. A. Rubakov, Int. J. Mod. Phys. A 7, 659 (1992).

[10] E. Shuster and D. T. Son, Nucl. Phys. B573, 434 (2000).

[11] B. Y. Park, M. Rho, A. Wirzba, and I. Zahed, Phys. Rev. D 62, 034015 (2000).

[12] M. Rozali, H. H. Shieh, M. Van Raamsdonk, and J. Wu, J. High Energy Phys. 01 (2008) 053.

[13] L. McLerran and R. D. Pisarski, Nucl. Phys. A796, 83 (2007).

[14] R. Rapp, E. V. Shuryak, and I. Zahed, Phys. Rev. D 63, 034008 (2001).

[15] M. Sadzikowski and W. Broniowski, Phys. Lett. B 488, 63 (2000).

[16] E. Nakano and T. Tatsumi, Phys. Rev. D 71, 114006 (2005).

[17] D. Nickel, Phys. Rev. Lett. 103, 072301 (2009).
[18] O. Schnetz, M. Thies, and K. Urlichs, Ann. Phys. (N.Y.) 314, 425 (2004).

[19] O. Schnetz, M. Thies, and K. Urlichs, Ann. Phys. (N.Y.) 321, 2604 (2006).

[20] M. Thies, J. Phys. A 39, 12707 (2006).

[21] F. Correa, G. V. Dunne, and M.S. Plyushchay, arXiv:0904.2768.

[22] G. Basar, G. V. Dunne, and M. Thies, Phys. Rev. D 79, 105012 (2009).

[23] W. Bietenholz, A. Gfeller, and U. J. Wiese, J. High Energy Phys. 10 (2003) 018.

[24] C. Itzykson and J. B. Zuber, International Series in Pure and Applied Physics (Mcgraw-Hill, New York, 1980), p. 705.

[25] G. Basar and G. V. Dunne, Phys. Rev. Lett. 100, 200404 (2008); Phys. Rev. D 78, 065022 (2008).

[26] S. P. Klevansky, Rev. Mod. Phys. 64, 649 (1992).

[27] A. I. Larkin and Yu. N. Ovchinnikov, Zh. Eksp. Teor. Fiz. 47, 1136 (1964) [Sov. Phys. JETP 20, 762 (1965)].

[28] J. A. Bowers and K. Rajagopal, Phys. Rev. D 66, 065002 (2002).

[29] C. Boehmer, M. Thies, and K. Urlichs, Phys. Rev. D 75, 105017 (2007).

[30] O. Scavenius, A. Mocsy, I. N. Mishustin, and D. H. Rischke, Phys. Rev. C 64, 045202 (2001).

[31] B. J. Schaefer and J. Wambach, Phys. Rev. D 75, 085015 (2007).

[32] D. Nickel and M. Buballa, Phys. Rev. D 79, 054009 (2009).

[33] B. J. Schaefer and J. Wambach, Nucl. Phys. A757, 479 (2005). 